\title{
EL ROL DEL TIPO DE CAMBIO EN PERU: ¿AMORTIGUADOR O FUENTE DE CHOQUES?
}

THE ROLE OF EXCHANGE RATE IN PERU: A SHOCK ABSORBER OR A SOURCE OF SHOCKS?

\section{JOAO RIBEIRO*}

Universidad Peruana de Ciencias Aplicadas

\begin{abstract}
Resumen
Este documento examina el rol del tipo de cambio en Perú utilizando un modelo estocástico para una economía pequeña y abierta que es identificado mediante un modelo estructural con vectores autorregresivos (SVAR) con restricciones de largo plazo a lo Blanchard y Quah. La descomposición de varianza indica que los choques reales de oferta y demanda son la principal fuente de fluctuaciones de los ciclos económicos en Perú y que conforme pasan los trimestres van ganando relevancia en explicar los movimientos del tipo de cambio. Estos resultados sugieren que el tipo de cambio juega un rol de absorber los choques reales que afectan la economía peruana.
\end{abstract}

Palabras clave: SVAR, macroeconomía abierta, tipo de cambio, Perú.

Clasificación JEL: C13, E44, E58, F41.

\begin{abstract}
This paper examines the role of the exchange rate in Peru using a stochastic model for a small open economy that is identified through a structural model with autoregressive vectors (SVAR) with long term restrictions to the Blanchard and Quah. The decomposition of variance indicates that the
\end{abstract}

* Correo: pcefjrib@upc.edu.pe; Universidad Peruana de Ciencias Aplicadas, Av. Primavera 2930, Monterrico, Santiago de Surco, Lima. Perú. E-mail: arturo.lorenzo@udlap.mx 
real supply and demand shocks are the main source of fluctuations in the economic cycles in Peru and that, as the quarters pass, they are gaining relevance in explaining the movements of the exchange rate. These results suggest that the exchange rate plays a role in absorbing the real shocks that affect the Peruvian economy.

Key words: SVAR, open macroeconomics, exchange rate, Peru.

JEL Classification: C13, E44, E58, F41.

\section{INTRODUCCION}

Las economías pequeñas y abiertas como la peruana están constantemente expuestas a choques externos e internos. Ante estas circunstancias, un manejo adecuado de la política cambiaria juega un rol importante para asimilar dichos choques. La teoría económica señala que, ante un tipo de cambio flexible, los choques de demanda tienen un efecto limitado en la inflación y el producto en el corto plazo, mientras que ocurre lo contrario con un tipo de cambio fijo. En vista que un régimen con tipo de cambio flexible promueve una política monetaria independiente, esto permite que las tasas de interés y el tipo de cambio absorban los choques de demanda. En este sentido, para economías que experimentan choques de demanda significativos y frecuentes puede ser más conveniente adoptar un esquema con tipo de cambio flexible que permita estabilizar la inflación y el producto.

En años recientes, varios estudios (An y Kim, 2010; Artis y Ehrmann, 2000; Bjornland, 2004; Clarida y Galí, 1994; Edwards y Yeyati, 2005; Farrant y Peersman, 2006; Funke, 2000; Goo y Siregar, 2009; Kim y Lee, 2008; Korhonen y Mehrotra, 2009; Thomas, 1997) han explorado el rol que juega la política cambiaria. En el caso de las economías asiáticas, An y Kim (2010), Goo y Siregar (2009), y Kim y Lee (2008) han estudiado el rol del tipo de cambio en Japón, Indonesia y Tailandia; y Corea del Sur, respectivamente. Estos estudios destacan que el tipo de cambio absorbe los choques en Japón, mientras que en Corea del Sur es una fuente de choques. En el caso de Indonesia y Tailandia, el tipo de cambio resulta ser una fuente de choques antes de la Crisis Asiática y un amortiguador de choques después de la misma.

Clarida y Galí (1994) analizan la relación entre el producto y el tipo de cambio real entre Estados Unidos, Japón, Alemania, Reino Unido y Canadá y encuentran que alrededor del $90 \%$ de las fluctuaciones del producto pueden ser atribuidas a choques reales y que cerca del $60 \%$ de los movimientos del tipo de cambio pueden ser atribuidos también a choques reales. Por su parte, Canzoneri, Valles, y Vinals (1996) también encuentran que los choques de oferta y demanda explican casi toda la variación del producto relativo entre Alemania y Austria, Holanda, Francia, Italia, España y Reino Unido. 
En este trabajo se utiliza un modelo macroeconómico estocástico para una economía abierta consistente con un modelo Estructural de Vectores Autorregresivos (SVAR). Se utiliza el modelo teórico para identificar los choques estructurales del SVAR por medio de la descomposición de Blanchard y Quah (1989). Después de identificar los cuatro choques del modelo (oferta, demanda, financiero y monetario), mediante el análisis de descomposición de varianza se encuentra que el tipo de cambio actúa absorbiendo choques reales a la economía peruana.

El resto del documento está organizado de la siguiente manera: en la Sección 2 se presenta una descripción del modelo teórico, mientras que en la Sección 3 se describe el modelo SVAR y en la Sección 4 se presentan los resultados. Finalmente, las conclusiones.

\section{MODELO MACROECONOMICO ESTOCASTICO PARA UNA ECONOMIA PEQUEÑA Y ABIERTA}

Como marco teórico se utiliza el modelo macroeconómico para una economía abierta desarrollado por Obstfeld (1985), y Clarida y Galí (1994). Se trata del modelo estocástico de dos economías con expectativas racionales y rigidez de precios. Sin embargo, el modelo es extendido para incluir choques financieros que son definidos como cambios inesperados en la prima de riesgo. Intuitivamente, esta modificación resulta importante debido a que los choques financieros son una posible fuente de fluctuaciones cambiarias, especialmente en economías pequeñas como la peruana. Además, como demuestra Dabrowski (2012), si los choques financieros no son identificados se pueden combinar con los choques de demanda y monetarios, lo que puede distorsionar los resultados empíricos. La segunda extensión del modelo es que todos los choques (no solo los de demanda) pueden tener componentes transitorios y permanentes.

El modelo consta de cuatro bloques, los que son las relaciones IS y LM, la condición de paridad descubierta de tasas de interés y la relación de fijación de precios:

$$
\begin{gathered}
y_{t}^{d}=d_{t}+\eta\left(s_{t}-p_{t}\right)-\sigma\left(i_{t}-E_{t}\left(p_{t+1}-p_{t}\right)\right) \\
m_{t}^{s}=p_{t}+y_{t}-\lambda i_{t} \\
i_{t}=E_{t}\left(s_{t+1}-s_{t}\right)+x_{t} \\
p_{t}=(1-\theta) E_{t-1} p_{t}^{e}+\theta p_{t}^{e}
\end{gathered}
$$

Todas las variables excepto las tasas de interés están expresadas en logaritmos y representan la diferencia entre los niveles domésticos $(\mathrm{H})$ y externos $(\mathrm{F})$ (por ejemplo, 
$p_{t}$ es definido como $p_{t}^{H}-p_{t}^{F}$ ). La Ecuación (1) es la relación IS que indica que la demanda relativa de bienes domésticos $y_{t}^{d}$ depende del choque $d_{t}$, el tipo de cambio real $q_{t}=s_{t}-p_{t}$ y el diferencial de tasas de interés reales $i_{t}-E_{t}\left(p_{t+1}-p_{t}\right)$. La Ecuación (2) es la condición de equilibrio en el mercado monetario (relación LM). La Ecuación (3) requiere que el diferencial de tasas de interés sea igual a la depreciación esperada de la moneda doméstica ajustada por una prima por riesgo $x_{t}$. La Ecuación (4) determina cómo se fijan los precios: dada la rigidez de precios, el índice de precios relativos $p_{t}$ es el promedio entre el nivel de equilibrio esperado en el período $t-1$ para el período $t$ y el nivel de precios que debe equilibrar el mercado ante precios totalmente flexibles en $t\left(p_{t}^{e}\right)$.

Entonces, el modelo presenta cuatro choques estocásticos que afectan el producto relativo $y^{s}$, la demanda relativa $d_{t}$, la cantidad relativa de dinero $m_{t}$ y la prima de riesgo $x_{t}$ :

$$
\begin{gathered}
y_{t}^{s}=y_{t-1}^{s}+\varepsilon_{t}^{s}-\gamma_{1} \varepsilon_{t-1}^{s} \\
d_{t}=d_{t-1}+\varepsilon_{t}^{d}-\gamma_{2} \varepsilon_{t-1}^{d} \\
x_{t}=x_{t-1}+\varepsilon_{t}^{x}-\gamma_{3} \varepsilon_{t-1}^{x} \\
m_{t}=m_{t-1}+\varepsilon_{t}^{m}-\gamma_{4} \varepsilon_{t-1}^{m}
\end{gathered}
$$

De esta manera, los cuatro choques presentan un componente permanente y otro transitorio: se espera que una fracción $\gamma$ de cualquier choque en el período $t$ se revierta en $t+1$.

Se puede demostrar que ante un tipo de cambio flexible los niveles del equilibrio de largo plazo con precios flexibles y expectativas racionales son:

$$
\begin{gathered}
y_{t}^{e}=y_{t}^{s} \\
r_{t}^{e}=x_{t}+\frac{1}{\eta+\sigma}\left(-\gamma_{1} \varepsilon_{t}^{s}+\gamma_{2} \varepsilon_{t}^{d}\right) \\
q_{t}^{e}=\frac{\sigma x_{t}-y_{t}^{s}-d_{t}}{\eta}+\frac{\sigma}{\eta(\eta+\sigma)}\left(-\gamma_{1} \varepsilon_{t}^{s}+\gamma_{2} \varepsilon_{t}^{d}-\sigma \gamma_{3} \varepsilon_{t}^{f}\right) \\
p_{t}^{e}=m_{t}-y_{t}^{s}+\lambda x_{t}+\alpha_{1} \gamma_{1} \varepsilon_{t}^{s}+\alpha_{2} \gamma_{2} \varepsilon_{t}^{d}-\alpha_{3} \gamma_{3} \varepsilon_{t}^{f}-\alpha_{4} \gamma_{4} \varepsilon_{t}^{m}
\end{gathered}
$$

donde $\alpha_{i}$ están en función de los parámetros y son de valor positivo. 


\section{ESTRATEGIA EMPIRICA Y DATOS}

\subsection{Metodología empírica}

Los principales resultados de las Ecuaciones (9)-(12) son utilizados como restricciones para identificar los choques y estimar la versión SVAR del modelo teórico. Utilizar un modelo VAR permite mayores relaciones dinámicas entre las variables al incluir rezagos en el sistema.

Además, estimar el modelo estocástico utilizando un SVAR con cuatro variables permite identificar empíricamente los cuatro choques estructurales. Se asume $x_{t}$ como un vector de procesos estacionarios en covarianza con una forma en promedio móviles de su forma SVAR con el vector de choques originales:

$$
x_{t}=D(L) \varepsilon_{t}
$$

donde $\mathrm{L}$ es el operador de rezagos y $\varepsilon_{t}=\left[\varepsilon_{t}^{s}, \varepsilon_{t}^{d}, \varepsilon_{t}^{x}, \varepsilon_{t}^{m}\right]$ es el vector de choques estructurales. Los choques estructurales no están correlacionados y tienen una matriz de varianzas-covarianzas normalizada a una matriz de identidad.

El vector de choques estructurales $\varepsilon_{t}$ directamente no es observable. Sin embargo, $\varepsilon_{t}$ puede ser identificado a partir de la estimación de la representación con promedios móviles de la forma reducida del VAR:

$$
x_{t}=C(L) u_{t}
$$

donde $u_{t}$ es el vector de residuos no correlacionados de la forma reducida con una matriz de covarianzas $\Omega$. Se asume que existe una matriz no singular $D_{0}$ tal que los choques de la forma reducida pueden ser escritos como una combinación lineal de los choques estructurales:

$$
u_{t}=D_{0} \varepsilon_{t}
$$

Es necesario identificar la matriz $D_{0}$ para que sea posible recuperar el vector de choques estructurales a partir de los residuos de la forma reducida. A partir de (14) y (15) se obtiene $C(L) D_{0}=D(L)$. Entonces, si $D_{0}$ es identificado, la representación de promedios móviles en (13) puede ser derivada.

Debido a que $\operatorname{var}\left(\varepsilon_{t}\right)$ es normalizada como una matriz de identidad, la matriz de covarianzas de los residuos puede ser definida como $\Omega=D_{0} D_{0}^{\prime}$. En este caso, para el sistema de cuatro variables la matriz simétrica $\Omega=D_{0} D_{0}^{\prime}$ impone 10 de las 16 restricciones en los elementos de $D_{0}$. Entonces, para poder identificar el modelo estructural se necesitan seis restricciones adicionales para identificar $D_{0}$. Estas restricciones se determinarán en los multiplicadores de largo plazo de la matriz $D(L)$. 
Blanchard y Quah (1989) y Clarida y Galí (1994) sugieren que estas restricciones de largo plazo estén basadas en la teoría económica tal que permita identificar los choques a base de la respuesta de las variables en el largo plazo. Así, Thomas (1997) indica que las restricciones de largo plazo ayudan a obtener una identificación adecuada de los choques.

La representación de largo plazo del sistema se puede escribir como:

$$
\left[\begin{array}{c}
y_{t}^{d} \\
r_{t} \\
s_{t} \\
p_{t}
\end{array}\right]=\left[\begin{array}{llll}
D_{11}(1) & D_{12}(1) & D_{13}(1) & D_{14}(1) \\
D_{21}(1) & D_{22}(1) & D_{23}(1) & D_{24}(1) \\
D_{31}(1) & D_{32}(1) & D_{33}(1) & D_{34}(1) \\
D_{41}(1) & D_{42}(1) & D_{43}(1) & D_{44}(1)
\end{array}\right]\left[\begin{array}{c}
\varepsilon_{t}^{s} \\
\varepsilon_{t}^{d} \\
\varepsilon_{t}^{f} \\
\varepsilon_{t}^{m}
\end{array}\right](16)
$$

donde $D(1)=\sum_{i=0}^{\infty} D_{i}$. El modelo teórico, descrito desde la ecuación (9) hasta la (12), sugiere que solo los choques de oferta tienen efecto sobre el producto en el largo plazo dado que $D_{12}(1)=D_{13}(1)=D_{14}(1)=0$. Así, después de imponer las seis restricciones de largo plazo, la $D(1)$ resulta como una matriz triangular inferior necesaria para recuperar $D_{0}$. A partir de $\Omega=D_{0} D_{0}^{\prime}$ y $C(L) D_{0}=D(L)$ se pueden determinar la siguiente expresión:

$$
D(1) D(1)^{\prime}=C(1) \Omega C(1)^{\prime}
$$

La ecuación (17) implica que dada la estimación de $C(1)$ y $\Omega, D(1)$ será el único factor triangular inferior a lo Cholesky de $C(1) \Omega C(1)^{\prime}$. Entonces, los pasos de estimación se resumen en: se estima la forma reducida del modelo VAR y se calcula $C(1)$ y $\Omega$, se utiliza (17) para obtener la matriz triangular inferior de Cholesky $D(1)$, y se calcula $D_{0}=C(1)^{-1} D(1)$. Una vez que se obtiene $D_{0}$ se pueden identificar los choques estructurales.

\subsection{Datos}

Se utiliza información trimestral desde el 2002 hasta el 2017. El PBI real es utilizado como medida de producto. La tasa de interés real es calculada como la diferencia entre la tasa de interés interbancaria y la tasa de inflación. El tipo de cambio real está en función del tipo de cambio nominal fin de período definido como la moneda extranjera (dólar) en función de la moneda local, tal que un incremento del tipo de cambio implica una depreciación de la moneda local. El nivel de precios es medido con el Índice de Precios al Consumidor. El producto relativo y el nivel de precios relativos son construidos como la diferencia en logaritmos de las variables domésticas y externas. El diferencial de las tasas de interés es la diferencia entre la tasa doméstica y la tasa externa medida con la tasa de los Fondos Federales de la Fed. 


\section{RESULTADOS}

\subsection{Modelo con dos variables}

En línea con Canzoneri, Vallés Liberal y Viñals (1996), se empieza el análisis empírico con el modelo VAR más parsimonioso posible que incluye solo dos variables: el producto relativo y el tipo de cambio. Esto permite identificar dos choques estructurales: no neutral o permanente $\varepsilon_{t}^{p}$ y neutral o transitorio $\varepsilon_{t}^{n}$, dependiendo de su impacto de largo plazo en el producto relativo. El modelo es

$$
\left[\begin{array}{c}
\Delta y_{t} \\
\Delta q_{t}
\end{array}\right]=\left[\begin{array}{cc}
D_{11}(L) & D_{12}(L) \\
D_{21}(L) & D_{22}(L)
\end{array}\right]\left[\begin{array}{c}
\varepsilon_{t}^{p} \\
\varepsilon_{t}^{n}
\end{array}\right]
$$

donde $D(L)$ son polinomios de rezagos y la restricción para la identificación es $D_{12}(1)=0$, es decir, los choques neutrales no tienen efecto de largo plazo sobre el producto.

\section{CUADRO 1}

DESCOMPOSICION DE VARIANZA DEL VAR CON DOS VARIABLES

\begin{tabular}{|c|c|c|c|}
\hline Variable & Período & Transitorio & Permanente \\
\hline \multirow{2}{*}{ Producto } & 1,0 & 75,7 & 24,3 \\
& 4,0 & 86,0 & 14,0 \\
\hline \multirow{2}{*}{ Tipo de cambio } & 1,0 & 29,1 & 70,9 \\
& 4,0 & 37,5 & 62,5 \\
\hline
\end{tabular}

Fuente: Estimaciones del autor.

Los resultados de la descomposición de varianza de ambas variables se describen en el Cuadro 1 y muestra que el choque que explica las fluctuaciones del producto es distinto del que determina los movimientos del tipo de cambio. El producto relativo registra una influencia bastante fuerte de los choques permanentes, los que llegan a explicar cerca del $90 \%$ de la varianza hacia un horizonte de un año. En cambio, poco más del $60 \%$ de las fluctuaciones del tipo de cambio son explicadas por los choques transitorios. Estos resultados en principio sugieren que el tipo de cambio es una fuente de choques para la economía. No obstante, la influencia alta y rápida de los choques transitorios sobre el tipo de cambio tiene un impacto limitado en el producto, mientras 
que el choque permanente del producto se va volviendo más importante en explicar los movimientos del tipo de cambio conforme pasan los trimestres. Así, el tipo de cambio como fuente de choques tiene un efecto transitorio y limitado sobre el producto, pero conforme los choques permanentes sobre el producto se van propagando el tipo de cambio empieza a ajustarse para ir absorbiendo parte del choque.

Es importante destacar que la forma en la que están definidos los choques puede generar problemas debido a que los choques transitorios pueden resultar una combinación de choques de demanda, financieros y monetarios. Así, Borghijs y Kuijs (2004) y Canzoneri et al. (1996) indican que la interpretación de los choques transitorios puede ser ambigua en el caso del VAR parsimonioso con dos variables.

\subsection{Modelo con tres variables}

Con la finalidad de distinguir los choques reales de demanda de los choques monetarios se utiliza un modelo VAR con tres variables. Siguiendo a Clarida y Galí (1994) se construye un modelo con el producto relativo, el tipo de cambio real y el nivel relativo de precios. En este caso, tres choques estructurales pueden ser identificados: oferta $\varepsilon_{t}^{s}$, demanda $\varepsilon_{t}^{d}$ y monetario $\varepsilon_{t}^{m}$. El modelo es

$$
\left[\begin{array}{c}
\Delta y_{t} \\
\Delta q_{t} \\
\Delta p_{t}
\end{array}\right]=\left[\begin{array}{ccc}
D_{11}(L) & D_{12}(L) & D_{13}(L) \\
D_{21}(L) & D_{22}(L) & D_{23}(L) \\
D_{31}(L) & D_{32}(L) & D_{33}(L)
\end{array}\right]\left[\begin{array}{c}
\varepsilon_{t}^{s} \\
\varepsilon_{t}^{d} \\
\varepsilon_{t}^{m}
\end{array}\right]
$$

donde las restricciones de identificación son $D_{12}(1)=D_{13}(1)=D_{23}(1)=0$, es decir, los choques de demanda no afectan el producto en el largo plazo y los choques monetarios son neutrales para el producto y el tipo de cambio. El Cuadro 2 muestra la descomposición de varianza con un horizonte de proyección de hasta un año.

Primero, más del $80 \%$ de las fluctuaciones del producto son explicadas por los choques de oferta. Segundo, cerca del $70 \%$ de las fluctuaciones cambiarias son explicadas principalmente por los choques de demanda, mientras que los choques monetarios explican menos del $1 \%$. Finalmente, los choques reales explican poco más del $30 \%$ de la varianza del nivel de precios. Entonces, se obtienen resultados similares a los encontrados con el modelo con dos variables que sugieren que el tipo de cambio juega un rol absorbiendo choques.

Si bien el VAR con tres variables da mayores indicios de los choques detrás de las fluctuaciones cambiarias, no se puede hacer la distinción entre choques financieros y monetarios. Sin embargo, el problema puede ser resuelto al introducir un choque financiero por medio de un modelo VAR de cuatro variables. 
CUADRO 2

DESCOMPOSICION DE VARIANZA DEL VAR CON TRES VARIABLES

\begin{tabular}{|l|c|c|c|c|}
\hline Variable & Período & Oferta & Demanda & Monetario \\
\hline \multirow{2}{*}{ Producto } & 1,0 & 66,5 & 24,0 & 9,4 \\
& 4,0 & 85,5 & 10,5 & 4,0 \\
\hline \multirow{2}{*}{ Tipo de cambio } & 1,0 & 26,5 & 72,8 & 0,7 \\
& 4,0 & 30,8 & 68,4 & 0,7 \\
\hline \multirow{2}{*}{ Precios } & 1,0 & 13,8 & 1,0 & 85,3 \\
& 4,0 & 15,4 & 8,3 & 76,4 \\
\hline
\end{tabular}

Fuente: Estimaciones del autor.

\subsection{Modelo con cuatro variables}

Con la finalidad de identificar los choques financieros, el modelo se extiende a cuatro variables. Así, se estima el modelo con cuatro variables: producto relativo, el diferencial de tasas de interés reales, el tipo de cambio y el nivel relativo de precios. Esto permite identificar cuatro choques estructurales: oferta $\varepsilon_{t}^{s}$, demanda $\varepsilon_{t}^{d}$, financiero $\varepsilon_{t}^{f}$ y monetario $\varepsilon_{t}^{m}$. El modelo es

$$
\left[\begin{array}{c}
\Delta y_{t} \\
\Delta r_{t} \\
\Delta q_{t} \\
\Delta p_{t}
\end{array}\right]=\left[\begin{array}{cccc}
D_{11}(L) & D_{12}(L) & D_{13}(L) & D_{14}(L) \\
D_{21}(L) & D_{22}(L) & D_{23}(L) & D_{24}(L) \\
D_{31}(L) & D_{32}(L) & D_{33}(L) & D_{34}(L) \\
D_{41}(L) & D_{42}(L) & D_{43}(L) & D_{44}(L)
\end{array}\right]\left[\begin{array}{c}
\varepsilon_{t}^{s} \\
\varepsilon_{t}^{d} \\
\varepsilon_{t}^{f} \\
\varepsilon_{t}^{m}
\end{array}\right](20)
$$

donde las restricciones de identificación son $D_{12}(1)=D_{13}(1)=D_{14}(1)=D_{23}(1)=$ $D_{24}(1)=D_{34}(1)=0$ de tal manera que los choques de demanda y financieros no afectan el producto en el largo plazo. Asimismo, los choques monetarios son neutrales al producto, el diferencial de tasas y el tipo de cambio.

La importancia de los choques se muestra en el Cuadro 3. La descomposición de varianza del producto es dominada por los choques de demanda que llegan a explicar hasta cerca del $70 \%$ de la varianza. Por su parte, alrededor del $60 \%$ de las fluctuaciones del tipo de cambio son influenciadas por los choques financieros. Sin embargo, los choques de oferta llegan a explicar cerca del $30 \%$ de la varianza del tipo de cambio. Los choques financieros explican simultáneamente los movimientos del tipo de cambio y el producto, pero el efecto sobre este último es bajo (14\%) y 
transitorio. En contraste, tanto los choques de oferta como de demanda, que son las principales fuentes de fluctuaciones del producto, van ganando importancia en explicar las variaciones del tipo de cambio conforme pasan los trimestres. Así, nuevamente se tiene evidencia de que el tipo de cambio juega un rol en absorber otro tipo de choques.

\section{CUADRO 3}

DESCOMPOSICION DE VARIANZA DEL VAR CON CUATRO VARIABLES

\begin{tabular}{|l|c|c|c|c|c|}
\hline \multicolumn{1}{|c|}{ Variable } & Período & Oferta & Demanda & Financiero & Monetario \\
\hline \multirow{2}{*}{ Producto } & 1,0 & 0,3 & 81,4 & 13,9 & 4,4 \\
& 4,0 & 24,1 & 66,5 & 7,2 & 2,2 \\
\hline \multirow{2}{*}{ Diferencial de tasas } & 1,0 & 79,2 & 1,0 & 12,6 & 7,1 \\
& 4,0 & 81,8 & 13,1 & 3,3 & 1,7 \\
\hline \multirow{2}{*}{ Tipo de cambio } & 1,0 & 26,7 & 9,2 & 63,7 & 0,4 \\
& 4,0 & 28,9 & 10,3 & 60,4 & 0,4 \\
\hline \multirow{2}{*}{ Precios } & 1,0 & 14,0 & 1,3 & 3,6 & 81,1 \\
& 4,0 & 13,4 & 9,8 & 8,1 & 68,8 \\
\hline
\end{tabular}

Fuente: Estimaciones del autor.

\section{CONCLUSIONES}

Este documento examina el rol que tiene el tipo de cambio en los ciclos económicos de la economía peruana. Para ello se estima un modelo SVAR con restricciones de largo plazo en función de un modelo teórico para una economía pequeña y abierta.

El análisis de descomposición de varianza muestra que pese a que los choques financieros determinan simultáneamente las fluctuaciones del producto (14\%) y del tipo de cambio (64\%), los choques reales (oferta y demanda) son la principal fuente de fluctuaciones de los ciclos económicos en Perú. Además, en la medida que pasan los trimestres los choques reales van ganando importancia en explicar los movimientos del tipo de cambio. Estos resultados sugieren que el tipo de cambio absorbe los choques reales que afectan la economía peruana y que como fuente de choques financieros su impacto es limitado.

\section{REFERENCIAS}

AN, L. y Y. KIM (2010). "Sources of exchange rate movement in Japan: Is the exchange rate a shock absorber or a source of shock?". Review of International Economics 18 (2), 265-276. 
ARTIS, M. y M. EHRMANN (2006). "The exchange rate - A shock absorber or source of shocks? A study of four economics", Journal of International Money and Finance 31, 42-59.

BJORNLAND, H. (2004). "The Role of the Exchange Rate as a Source of Absorber in a Small Open Economy", Open Economies Review 15, 23-43.

BLANCHARD, O. y D. QUAH (1989). "The Dynamic Effects of Aggregate Demand and Supply Disturbances", American Economic Review 79, 655-673.

BLUEDORN, J. y C. BOWDLER (2010). "The Empirics of International Monetary Transmission: Identification and the Impossible Trinity", IMF Working Paper 15/79.

BORGHIS, A. y L. KUIJS (2004). "Exchange Rates in Central Europe: A Blessing or a Curse?", IMF Working Paper WP/04/2, 1-28.

CANZONERI, M., J. VALLES y J. VIÑALS (1996). "Do exchange rates move to address international macroeconomic imbalances?", CEPR Discussion Paper 1498.

CLARIDA R. y J. GALÍ (1994). "Sources of Real Exchange Rate Fluctuations: How Important Are Nominal Shocks?", Carnegie-Rochester Conference Series on Public Policy 41, 1-56.

DABROWSKI, M. (2012a). "Exchange Rate Regimes and Output Variability in Central European Countries", Actual Problems of Economics 2/10, 80-91.

DABROWSKI, M. y J. WRÓBLEWWSKA (2015). "Exchange Rate as a Shock Absorber or a Shock Propagator in Poland and Slovakia: An Approach Based on Bayesian SVAR Models with Common Serial Correlation", MPRA Paper $\mathrm{N}^{\circ} 61441$.

DOOJAV, G. (2011). "The Role of Exchange Rate in Mongolia: A Shock Absorber or a Source of Shocks?", MPRA Paper $\mathrm{N}^{\circ} 72145$.

EDWARDS, S. y E. YEYATI (2005). "Flexible Exchange Rates as Shock Absorbers", European Economic Review 49 (2005), 2079-2105.

FARRANT, K. y G. PEERSMAN (2006). "Is the Exchage Rate a Shock Absorber or a Source of Shocks? New Empirical Evidence", Journal of Money, Credit, and Banking 38 (4), 939-961.

FUNKE, M. (2000). "Macroeconomic Shocks in Euroland vs. The UK: Supply, Demand or Nominal?", Department of Economics, Hamburg University, Hamburg, July 2000.

GOO, S. y R. SIREGAR (2009). "Economic Shocks and Exchange Rate as a Shock Absorber in Indonesia and Thailand", SEACEN Centre, Staff Paper $N^{\circ} 72$.

KIM, S. y S. LEE (2008). "The Role of the Exchange Rate in Korean Economy: A Shock Absorber or a Source of Shocks", Journal of Economic Research 13, 303-324.

KORHONEN, I. y A. MEHROTRA (2009). "Real Exchange Rate, Output and Oil: Case of Four Large Energy Producers", BOFIT Discussion Papers 6/2009.

OBSTFELD, M. (1985). "Floating Exchange Rates: Experience and Prospects", Brooking Papers on Economic Activity 2, 369-464.

THOMAS, A. (1997). "Is the Exchange Rate a Shock Absorber? The Case of Sweden", IMF Working Paper 97/176. 
